\title{
Korrespondenzen.
}

\section{Bemerkungen zu der Arbeit von Strubell „Ueber die Wrightsche Vakzine-Therapie" in No. 6 dieser Wochen- schrift.}

Von Dr. J. P. zum Busch in London.

Wenn der Verfasser glaubt, daß man bisher „einwandfreie Staphylokokkenvakzine von einem bestimmten Standard bei absoluter Sterilität in genügenden Mengen" nicht habe beziehen können, so irrt er sich. Sowohl das Lister Institnte in London wie das Incorporated Liverpool Institute of Comparative Pathology und das Laboratorium von Burrongts, Wellcome \& Co. (um nur drei der bekanntesten Firmen zu nennen) bringen die verschiedensten Vakzinen in den Handel; vom Lister Institute habe ich sie selbst schon im Jahre 1907 bezogen. $\mathrm{Zu}$ haben sind Staphylokokkenvakzine (anreus und gemischte), Streptokokken-, Pneumokokken- und Gonokokkenvakzine, alle diese in drei verschiedenen Stärken; außerdem Cholera-, Typhns- und andere Vakzinen. Die meisten Apotheken halten dieso Vakzinen vorrätig, die des Lister Institute sind z. B. Tag und Nacht durch Allan and Hanburys zu beziehen.

Is ist ferner durchaus nichts Neues, derartige Impfungen, und zwar besonders bei Akne, Furunkulose etc., ohne Kontrolle des opsonischen Index zu machen; nicht nur W right nnd seine Schüler, sondern zahlreiche englische Aerzte tun dies seit längerer Zeit, und es finden sich darauf bezügliche Anmerkungen in mehr wie einer Arbeit, die sich mit der Vakzinetherapie befaßt.

Vor allem aber möchte ich die Leser dieser Wochenschrift davor warnen, mit allzu hochgespannten Erwartungen an die Vakzinetherapie heranzutreten. Wer die ganze Bewegung von Anfang an genau und mit kritischen Augen verfolgt hat, kann sich der Ueberzeugung kaum verschließen, daß die Arbeiten Wrights und seiner Schüler zahlreiche Widersprüche enthalten und vor allem einen großen Mangel an klinischer Erfahrung erkennen lassen, was ja auch bei Männern, die sich nur unit bakteriologischen Forschungen beschäftigen, kaum zu verwundern ist. Ich habe zahlreiche Fälle (besonders von Staphylokokkenerkrankungen) selbst behandelt und auch viele Fälle gesehen, die von Opsoninspezialisten behandelt worden waren, und zwar mit und ohne Kontrolle des Index. Viel Nutzen habe ich bisher leider von dieser Behandlung nicht gesehen, und es stimmt dies durchaus mit den Erfahrungen überein, die andere Beobachter gemacht haben. Es ist nicht immer genügend, nur die Literatur durchzulesen, es ist manchmal wichtiger, gesprächsweise zu erfahren, was bekannte Kliniker über eine Methode denken, und da will es mir doch scheinen, als sei man in London heute wesentlich weniger hoffnungsvoll als vor ein oder zwei Jahren. So erfreulich es wäre, wenn z. B. mehrere große Kliniken in Deutschland die Vakzinetherapie gründlich studieren nnd ihre Erfolge nach Jahresfrist veröffentlichen wollten, so wenig geeignet scheint es mir zu sein, schon heute diese Methode in die allgemeine Praxis einzuführen. 